Methodology All patients with early-stage CC (FIGO IA1-IIA1) undergoing robot-assisted radical hysterectomy in Spain and Portugal from 2009 to 2018 were included. Those centres with $>15$ cases were selected. Centres with recurrence rate $<10 \%$ were gathered in group $\mathrm{A}$ an those $\geq 10 \%$ in group B. The primary objective was to compare the oncological outcomes between groups after balancing by Propensity Score [PS] analysis. The groups were balanced in age, BMI, histology, Size, tumoral grade, ILV and adjuvant treatment. Second primary objective was to audit the pre-surgical quality indicators [QI] proposed by ESGO.

Result(s)* A total of 118 and 97 patients were well balanced (p-value 0.9483) between groups. 5 (4.3\%) vs 19 (19.6\%) recurrences occurred in group A vs group B, OR 1.23; $(95 \%$ CI, 1,13-1,35) p-value of 0.001 after a median follow-up of 51 months. Overall mortality and disease-specific mortality were significant higher in group B, OR 1.07; (95\% CI, 1.001.15 ) and 1.09; (95\% CI, 1.02-1.16) respectively (figure 1). Five of eight Q.I were fulfilled by both groups. Lower rates of pre-operative assessment with M.R.I was observed in group B. $1(20 \%)$ and $8(42 \%)$ recurrences were observed during the first two years of robotic experience in group A and B (figure 2). Intraoperative and postoperative complications occurred in 0.8 vs $6.2 \%$ (p 0.028 ) and $5.1 \%$ vs $12.4 \%$ (p $0.055)$ in groups $A$ and $B$ respectively.

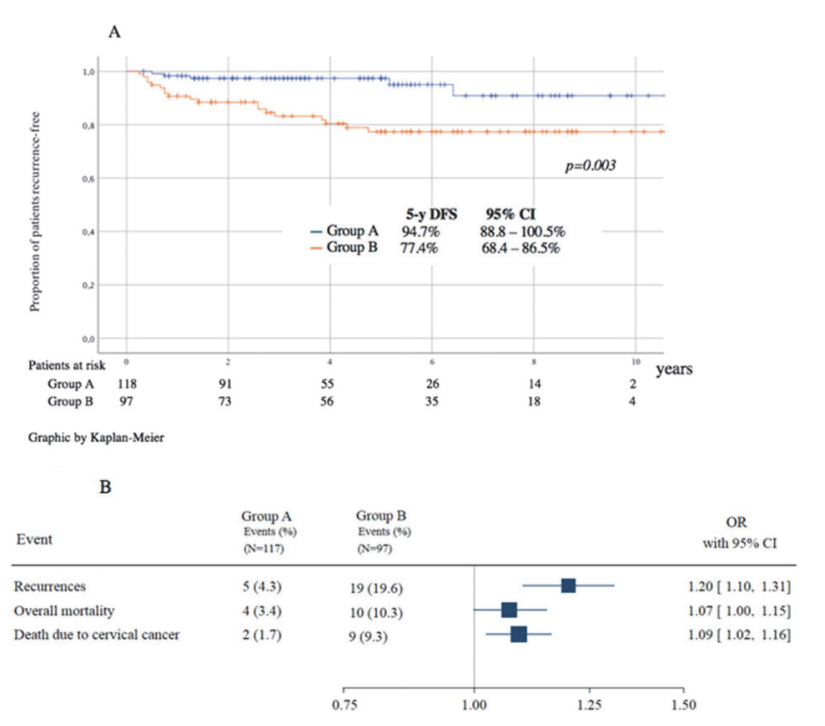

Abstract 245 Figure 1 A) Disease-free survival rates between surgical; B) Odds of recurrence \& mortality after balancing
Conclusion* We observed significant differences in recurrence rate, overall mortality and specific-disease mortality between hospitals. Pre-operative assessment with M.R.I and the effect of learning curve were factors related to higher rates of recurrence. The surgical assessment might be considered as an impact factor in oncological outcomes in patients who underwent radical hysterectomy by minimal invasive approach.

\section{ENGOT-CX11/GOG 3047/KEYNOTE-A18: PHASE 3 RANDOMIZED STUDY OF PEMBROLIZUMAB + CHEMORADIOTHERAPY FOR HIGH-RISK LOCALLY ADVANCED CERVICAL CANCER}

${ }^{1} \mathrm{D}$ Lorusso* ${ }^{2} Y$ Xiang, ${ }^{3} \mathrm{~N}$ Colombo, ${ }^{4} \mathrm{RL}$ Coleman, ${ }^{5} \mathrm{LM}$ Randall, ${ }^{6} \mathrm{~L}$ Duska, ${ }^{7} \mathrm{~K}$ Hasegawa, ${ }^{8} \mathrm{~A}$ Nogueira-Rodrigues, ${ }^{9} \mathrm{D}$ Cibula, ${ }^{10} \mathrm{MR}$ Mirza, ${ }^{11} \mathrm{~B}$ You, ${ }^{12} \mathrm{~A}$ Oaknin, ${ }^{13} \mathrm{M}$ Christiaens, ${ }^{14} \mathrm{C}$ Taskiran, ${ }^{15} \mathrm{~J}$ Sehouli, ${ }^{16} \mathrm{~J}$ Korach, ${ }^{17} \mathrm{C}$ Marth, ${ }^{18} \mathrm{~K}$ Yamada, ${ }^{18} \mathrm{M}$ Puglisi, ${ }^{19} \mathrm{~S}$ Pignata. ${ }^{1}$ Associate Professor of Obstetrics and Gynecology, Catholic University of Sacred Heart; Clinical Research Development Unit Fondazione Policlinico Gemelli IRCCS, Rome, Italy; ${ }^{2}$ Department of Gynecology and Obstetrics, Peking Union Medical College Hospital, Chinese Academy of Medical Sciences, Beijing, China; ${ }^{3}$ Department of Gynecology, Instituto Europeo di Oncologia, Milan, Italy; ${ }^{4}$ Department of Gynecologic Oncology and Reproductive Medicine, University of Texas MD Anderson Cancer Center, Houston, TX, USA; ${ }^{5}$ Department of Obstetrics and Gynecology, Massey Cancer Center, Virginia Commonwealth University, Richmond, VA, USA; ${ }^{6}$ Department of Obstetrics and Gynecology, University of Virginia, Charlottesville, VA, USA; ${ }^{7}$ Department of Gynecologic Oncology, Saitama Medical University, Hidaka, Saitama Prefecture, Japan; ${ }^{8}$ Departamento de Clínica Médica, Universidade Federal de Minas Gerais, Belo Horizonte, Minas Gerais, Brazil; ${ }^{9}$ Department of Obstetrics and Gynecology, General Faculty Hospital in Prague, First Faculty of Medicine, Charles University, Prague, Czech Republic; ${ }^{10}$ Department of Oncology, Copenhagen University Hospital, Copenhagen, Denmark; ${ }^{11}$ Department of Medical Oncology, CITOHL, IC-HCL, Hospices Civils de Lyon, Université Claude Bernard Lyon 1, Lyon, France; ${ }^{12}$ Department of Medical Oncology, Vall d'Hebron Institute of Oncology (VHIO), Hospital Universitari Vall d'Hebron, Vall d'Hebron Barcelona Hospital Campus, Barcelona, Spain; ${ }^{13}$ Department of Radiation Oncology, Universitair Ziekenhuis Leuven, Leuven, Belgium; ${ }^{14}$ Professor Koc University School of Medicine and VKV American Hospital, Department of Obstetrics and Gynecology, Division of Gynecologic Oncology and Turkish Society of Gynecologic Oncology, Istanbul, Turkey; ${ }^{15}$ Deparment of Gynecology, Charité-Universitätsmedizin Berlin, Berlin, Germany; ${ }^{16}$ Gynecology Oncology Department, Sheba Medical Center, Ramat Gan, Israel; ${ }^{17}$ Department of Obstetrics and Gynecology, Medizinische Universität Innsbruck, Innsbruck, Austria; ${ }^{18}$ Clinical Development, Merck and Co., Inc., Kenilworth, NJ, USA; ${ }^{19}$ Department of Uro-Gynaecological Oncology, Instituto Nazionale Tumori IRCCS Fondazione G Pascale, Italy

\subsection{6/ijgc-2021-ESG0.21}

Introduction/Background* High-risk locally advanced cervical cancer has a poor prognosis, and more than half of patients recur in 2 years. External beam radiotherapy (EBRT) with concurrent chemotherapy followed by brachytherapy is the standard of care for locally advanced cervical cancer. The

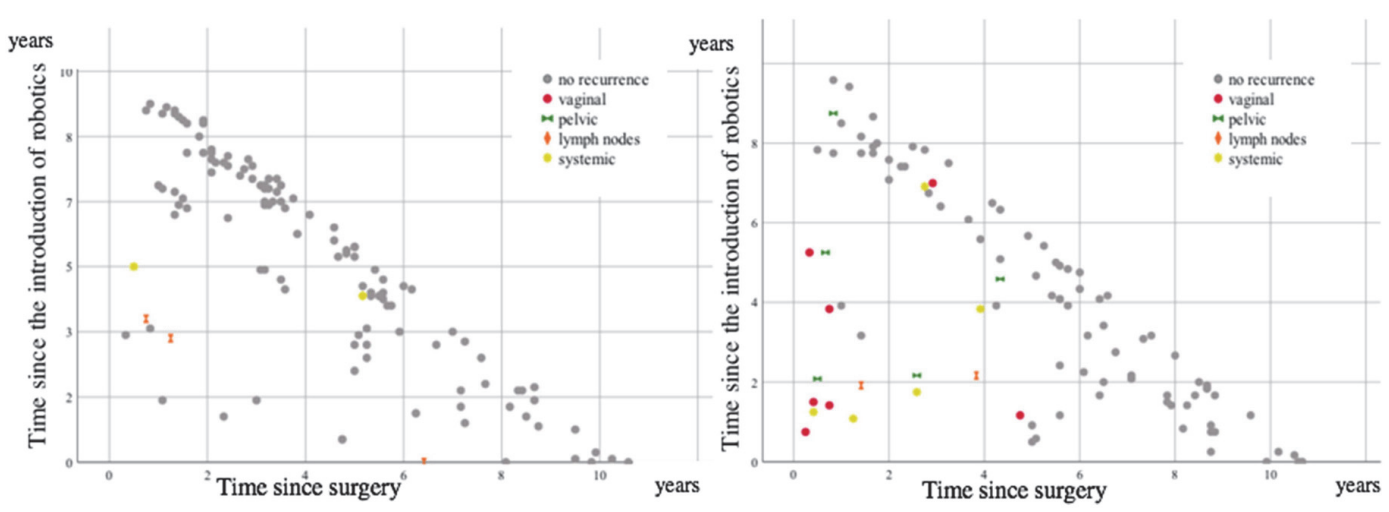

Abstract 245 Figure 2 (Group A and B) relation between time of recurrence and time of surgery 
immunostimulatory activity of the PD-1 inhibitor pembrolizumab may be enhanced by concurrent chemoradiotherapy (CCRT). After the KEYNOTE-158 study, in which pembrolizumab showed durable antitumor activity, pembrolizumab monotherapy was approved for patients with PD-L1-positive recurrent or metastatic cervical cancer who progressed during or after chemotherapy. ENGOT-cx11/GOG 3047/KEYNOTEA18 (NCT04221945) is a phase 3, randomized, placebo-controlled study evaluating pembrolizumab with CCRT for the treatment of high-risk, locally advanced cervical cancer.

Methodology Approximately 980 patients with high-risk (International Federation of Gynecology and Obstetrics 2014 stage IB2-IIB with node-positive disease or stage IIIIVA), locally advanced, histologically confirmed cervical cancer who have not received systemic therapy, immunotherapy, definitive surgery, or radiation will be randomized 1:1 to receive either 5 cycles of pembrolizumab $200 \mathrm{mg}$ every 3 weeks $(\mathrm{Q} 3 \mathrm{~W})$ + CCRT followed by 15 cycles of pembrolizumab $400 \mathrm{mg}$ Q6W or 5 cycles of placebo Q3W + CCRT followed by 15 cycles of placebo Q6W. CCRT includes 5 cycles (optional 6th dose) of cisplatin $40 \mathrm{mg} / \mathrm{m}^{2} \mathrm{Q} 1 \mathrm{~W}+$ EBRT followed by brachytherapy. Randomization is stratified by planned EBRT type (intensity-modulated radiotherapy [IMRT] or volumetric-modulated arc therapy [VMAT] vs non-IMRT or non-VMAT), cancer stage at screening (stage IB2-IIB vs III-IVA), and planned total radiotherapy dose. Treatment will continue until the patient has received 20 cycles of pembrolizumab (5 cycles $200 \mathrm{mg}$ Q3W, 15 cycles $400 \mathrm{mg}$ Q6W) vs placebo ( 2 years) or until disease progression, unacceptable toxicity, or withdrawal. Primary endpoints are progression-free survival (PFS) per Response Evaluation Criteria in Solid Tumors version 1.1 by investigator and overall survival (OS). Secondary endpoints include PFS by blinded independent central review, PFS at 2 years, OS at 3 years, complete response at 12 weeks, objective response rate, PFS and OS by PD-L1 status, quality of life, and safety. Enrolment began May 2020 and is planned for 193 sites in 30 countries.

Klikně te nebo klepně te sem a zadejte text.

\section{MRI BASED TUMOUR RADIOMICS IN CARCINOMA CERVIX: A POTENTIAL PREDICTOR OF RESPONSE TO CHEMORADIATION}

'S Chopra, ${ }^{2} \mathrm{P}$ Nayak, 'Y Gupta, 'I Swamidas, 'IS Goda, 'S Panda, 'L Gurram, '3 Rath, ${ }^{4} \mathrm{P}$ Popat, ${ }^{2} \mathrm{U}$ Mahantshetty, ${ }^{3} \mathrm{~S}$ Gupta, ${ }^{2} \mathrm{JP}$ Agarwal. 'ACTREC, Tata Memorial Centre, Radiation Oncology, Navi Mumbai, India; ${ }^{2}$ Tata Memorial Hospital, Tata Memorial Centre, Radiation Oncology, Mumbai, India; ${ }^{3} T a t a$ Memorial Hospital, Tata Memorial Centre, Medical Oncology, India; ${ }^{4}$ Tata Memorial Hospital, Tata Memorial Centre, Radiology, India

\subsection{6/ijgc-2021-ESGO.22}

Introduction/Background* To determine if baseline T2weighted (T2W) MRI texture parameters can predict response to chemoradiation in cervical carcinoma.

Methodology Seventy-four patients of locally advanced carcinoma cervix treated with definitive chemoradiation (45Gy in 25 fractions and weekly cisplatin $\left(40 \mathrm{mg} / \mathrm{m}^{2}\right)$ and image guided brachytherapy between 2017 and 2019 were included. Gross tumour volume (GTV) and high risk clinical target volume (HRCTV) was delineated on T2W MRI at baseline and at brachytherapy using the texture analysis software. Tumour regression $>75 \%$ was considered as a surrogate of good response. Multiple tumour slices were sampled and first order

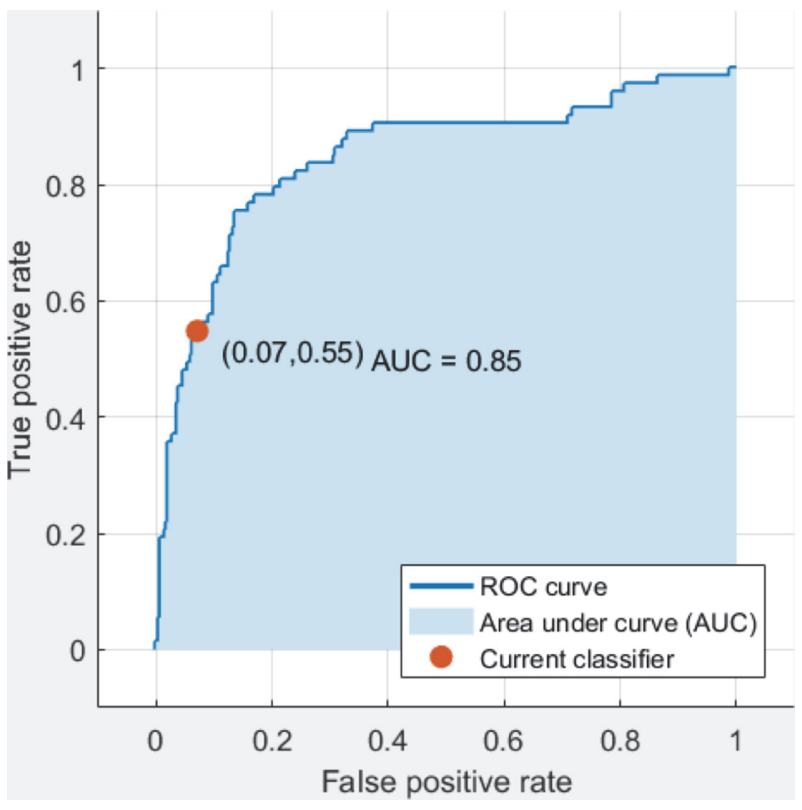

\section{Abstract 262 Figure 1}

statistics were applied to produce 6 texture parameters, namely mean intensity, standard deviation, entropy, mean positive pixels, skewness, and kurtosis. After using fine,moderate, and coarse anatomical filters a total of 36 texture variables were generated.

Clinical variables namely histological subtype, tumour grade, FIGO 2009 stage and nodal status were documented. These clinical variables along with texture parameters were compared with treatment response using the Mann-Whitney U test. Lasso regression was used to select texture parameters that best correlated with treatment response. These were used to develop support vector machine (SVM) models which were validated using 10-fold cross validation. The most parsimonious model was described in terms of area under curve (AUC) and metrics of diagnostic accuracy.

Result(s)* The median age was 50 years (range 34 to 65). Overall, $63(85 \%)$ had squamous cell carcinoma. Half of the included patients $(37 / 74)$ had positive pelvic or para-aortic lymph nodes. As per FIGO 2018 criteria 23 (31\%) and 51 (69\%) patients were stage II and III, respectively. Good response was seen in $10 / 74$ patients. None of the clinical variables discriminated between response. However, high mean and skewness, and low entropy and kurtosis did significantly correlate with poor response. Using 15 selected features, the best SVM model had an AUC of 0.85 and accurately classified $86.5 \%$ cases, with sensitivity and specificity of $55 \%$ and $93 \%$, respectively.

Conclusion* Tumour radiomics can be utilized to predict response to chemoradiation whereas baseline clinical parameters do not predict for response. Baseline texture analysis can be an important tool to predict response to chemoradiation.

\section{SOCIOECONOMIC FACTORS ASSOCIATED WITH PREVALENCE AND STAGING OF CERVICAL CANCER}

MG Theresa*, FW Wagey, BJ Laihad, FM Wagey. Manado, Obstetric and Gynecology Prof. Dr. R. D. Kandou General Hospital, Manado, Manado, Indonesia

10.1136/ijgc-2021-ESGO.23 